\title{
Mediastinal Small Cell Carcinoma with Primary Cutaneous Cryptococcosis: A Rare Case Report
}

\author{
Mengjie $\mathrm{Hu}$ (D) $^{1} * *$ \\ Songling $\mathrm{Chai}^{2, *}$ \\ Wenyi Lei (1D' \\ Kexin Liao' \\ Rongxin Zhang' \\ 'Department of Dermatology, The \\ Second Hospital of Dalian Medical \\ University, Dalian, Liaoning, People's \\ Republic of China; ${ }^{2}$ Department of Oral \\ and Maxillofacial Surgery, Stomatology \\ College of Dalian Medical University, \\ Dalian, Liaoning, People's Republic of \\ China
}

*These authors contributed equally to this work
Correspondence: Rongxin Zhang Department of Dermatology, The Second Hospital of Dalian Medical University, No. 467 Zhongshan Road, Dalian, I I6027, Liaoning, People's Republic of China

Tel +8617709873080

Fax $+86-4$ II-84672I30

Email zrx_1980@I26.com

\begin{abstract}
Cutaneous cryptococcosis, an infectious disease resulting from Cryptococcus neoformans, primarily affects immunodeficient individuals. Here, we report a case of mediastinal small cell carcinoma (MSCC) complicated with multiple skin and soft tissue infections mimicking erysipelas and cellulitis. Antibiotics for bacteria were ineffective and a culture of pus from the infected areas revealed Cryptococcus neoformans in this patient. The absence of any evidence indicative of systemic cryptococcal infection leads to a final diagnosis of primary cutaneous cryptococcosis (PCC). Following two weeks of fluconazole at $400 \mathrm{mg}$ /day and $200 \mathrm{mg}$ /day for the subsequent three months, combined with incision, irrigation and drainage, the wound gradually healed. An analysis and discussion of the clinical features of this patient are presented. This case alerts clinicians as to the possibility of Cryptococcus neoformans in patients with advanced malignant tumors complicated with multiple skin and soft tissue infections. While a timely diagnosis and treatment of PCC in this patient resulted in a favorable outcome, the patient succumbed to the malignant tumor at six months post-discharge.
\end{abstract}

Keywords: mediastinal small cell carcinoma, primary cutaneous cryptococcosis, Cryptococcus neoformans, immunodeficiency, therapy

\section{Introduction}

Cryptococcosis is a chronic, subacute or even acute infection of the brain, meninges, lungs, skin or entire body resulting from Cryptococcus infection. Cryptococcus neoformans, an opportunistic pathogen mainly infecting immunocompromised hosts, invades the central nervous system and lungs. ${ }^{1-3}$ Cutaneous infections are the third most common clinical manifestation of cryptococcosis, and these patients can present with various skin aberrations such as, papules, nodules, tumors, ulcerations, ecchymoses, pustules, abscesses and granulomas. ${ }^{4-7}$ Early identification of polymorphic skin manifestations and treatment are essential for an effective prognosis of the infection. This condition can be divided into primary and secondary cutaneous cryptococcosis. Primary cutaneous cryptococcosis (PCC), which is rare, but life-threatening, was first recognized in 2003. It mainly results from direct infection of skin trauma with no apparent symptoms of systemic disease. $^{8}$ Secondary cutaneous cryptococcosis, a more common type, mainly spreads from the brain, lung and other parts of the body. Immunodeficient individuals, as can result from AIDS, malignant tumors, organ transplantation and treatment with corticosteroids and immunosuppressive agents, represent the most susceptible cohort for cutaneous cryptococcosis. ${ }^{9}$ 
Mediastinal small cell carcinoma (MSCC), a rare tumor with a poor prognosis, is characterized by lesions located in the mediastinum. To the best of our knowledge, no more than 20 case reports of MSCC have been published in the literature. ${ }^{10}$ Here, we describe a rare, previously unreported case of PCC in a woman with MSCC.

\section{Case Presentation}

A 53-year-old female presented with multiple painful skin lesions on both thighs, without chills and fever. She had been treated with cephalosporin antibiotics prior to admission, however, her condition was not relieved and gradually aggravated. The patient had a history of MSCC for two years and received surgical treatment for this condition followed by four cycles of chemotherapy with irinotecan and cisplatin. Chemotherapy was eventually abandoned due to bone marrow suppression and, after one year, multiple metastases of the tumor were present throughout her entire body. She had no history of human immunodeficiency virus (HIV) infection and no contact with pigeons, poultry or other types of animals. Physical examination indicated painful skin lesions with erythema, swelling and abscesses on both thighs (Figure 1A-D). Laboratory assay results revealed that carbohydrate antigen 125 (CA125), CA199 and neuron-specific enolase (NSE) were substantially increased, while results of her computed tomography (CT) scan of the chest and abdomen showed multiple metastases of the tumor within the lung, liver, left adrenal gland, retroperitoneum, vertebral body and caput femoris (Figure 2AD). Bacterial culture of the abscess puncture fluid was negative, but fungal culture and antifungal susceptibility tests revealed the growth of Cryptococcus and its sensitisation to fluconazole. Blood and cerebrospinal fluid (CSF) cultures, cryptococcal antigen and serological tests were negative. No abnormalities were observed for other test results, such as transthoracic echocardiography (TTE), electrocardiogram (ECG), CT of brain and HIV infection. The diagnosis was PCC based on these clinical features and results of the pus fungal culture. Treatment with fluconazole (400 mg/day) via intravenous infusion was then initiated and the abscess cavities were rinsed with fluconazole saline solution twice daily after local incision and drainage (Figure 3A and B). In addition, the patient received symptomatic and supportive treatment. After a week of this treatment, inflammation within the skin lesions was clearly improved (Figure 3C and D), but wound healing was very slow due to the cachexia resulting from the advanced stage of the tumor. The fluconazole dose was then changed to an oral administration of $200 \mathrm{mg} /$ day for the following three months after the initial two-week intravenous infusion. Follow-up of the patient indicated no recurrence of the skin lesions. Unfortunately, she died six months postdischarge as a result of multiple organ failure from tumor metastases.
A

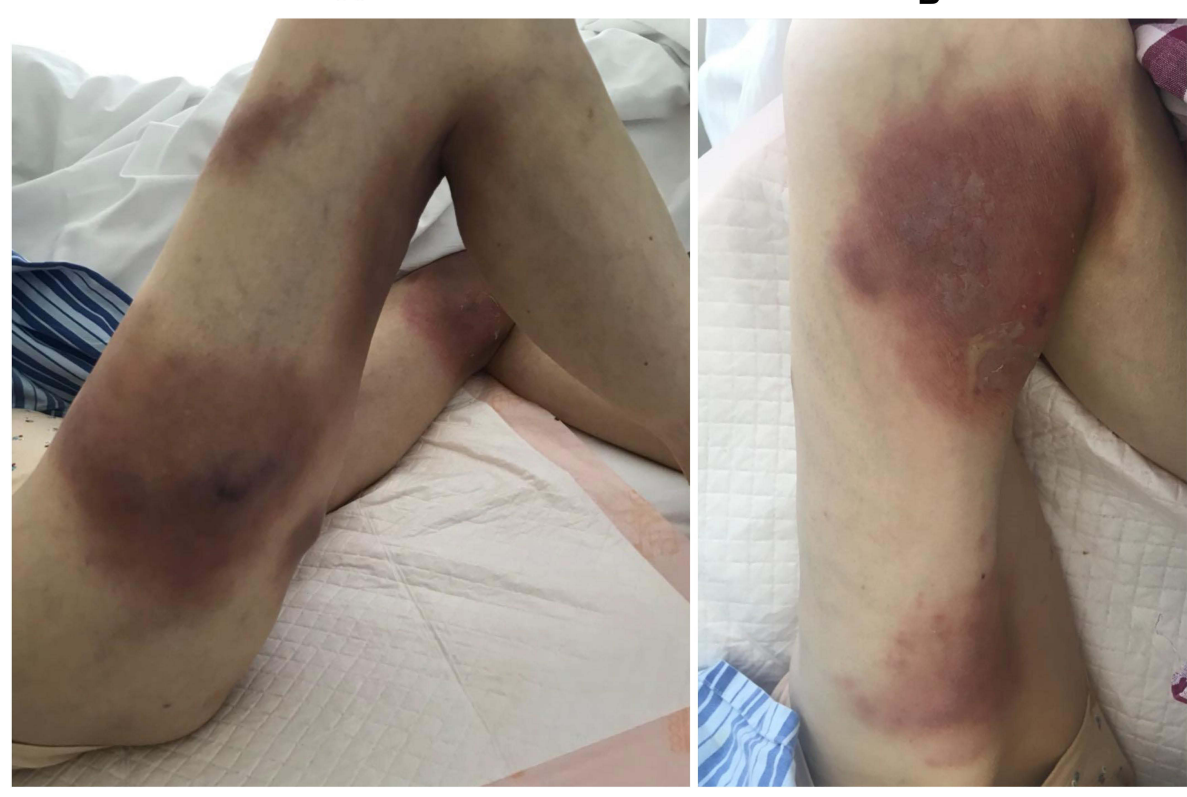

B

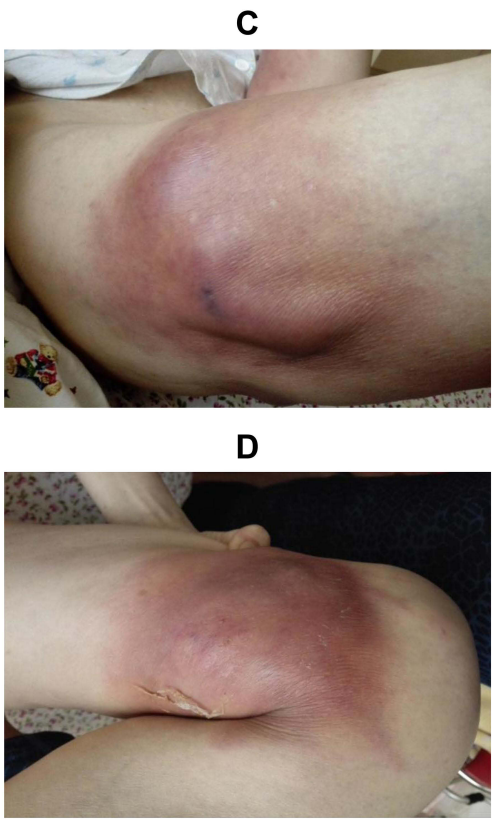

Figure I Physical examination of the female patient diagnosed as mediastinal small cell carcinoma complicated with primary cutaneous cryptococcosis. Multiple painful skin lesions with erythema and swelling on both thighs (A and $\mathbf{B})$. Abscesses on both thighs (C and $\mathbf{D})$. 
A
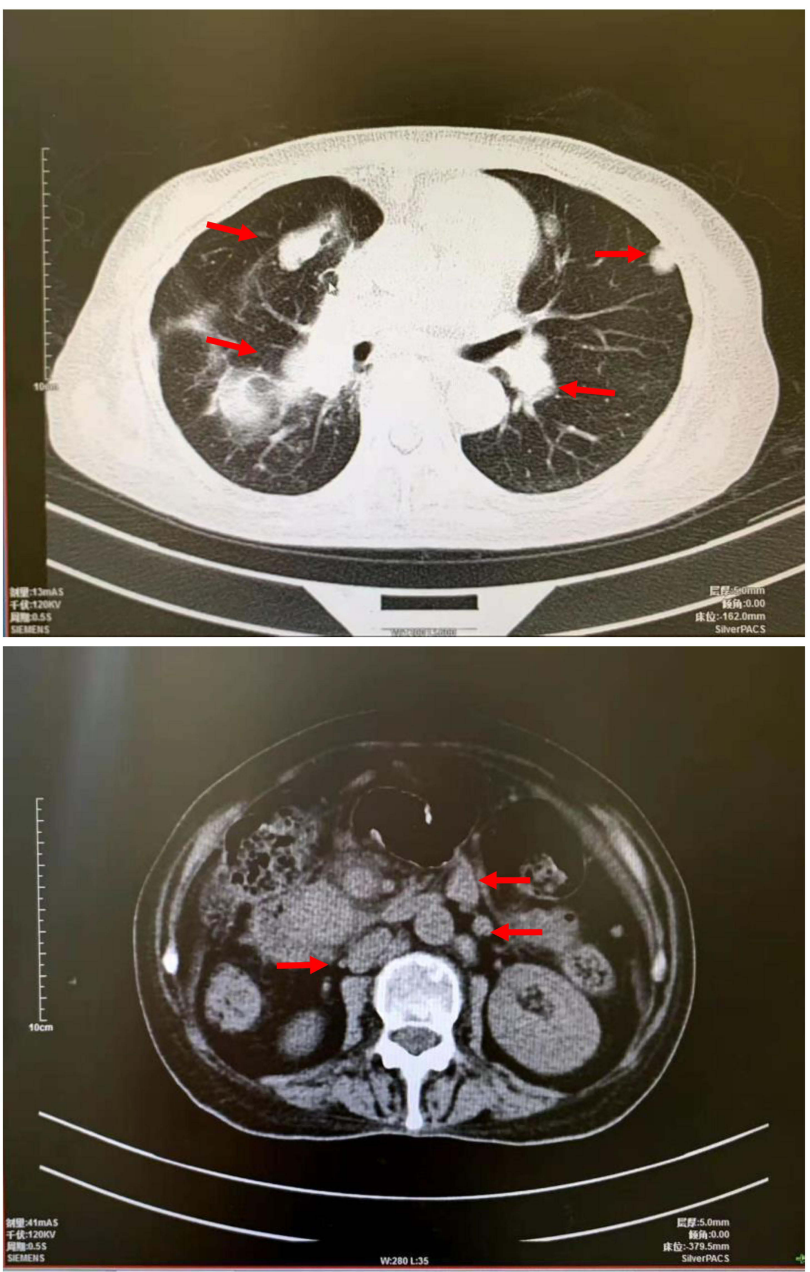

C
B
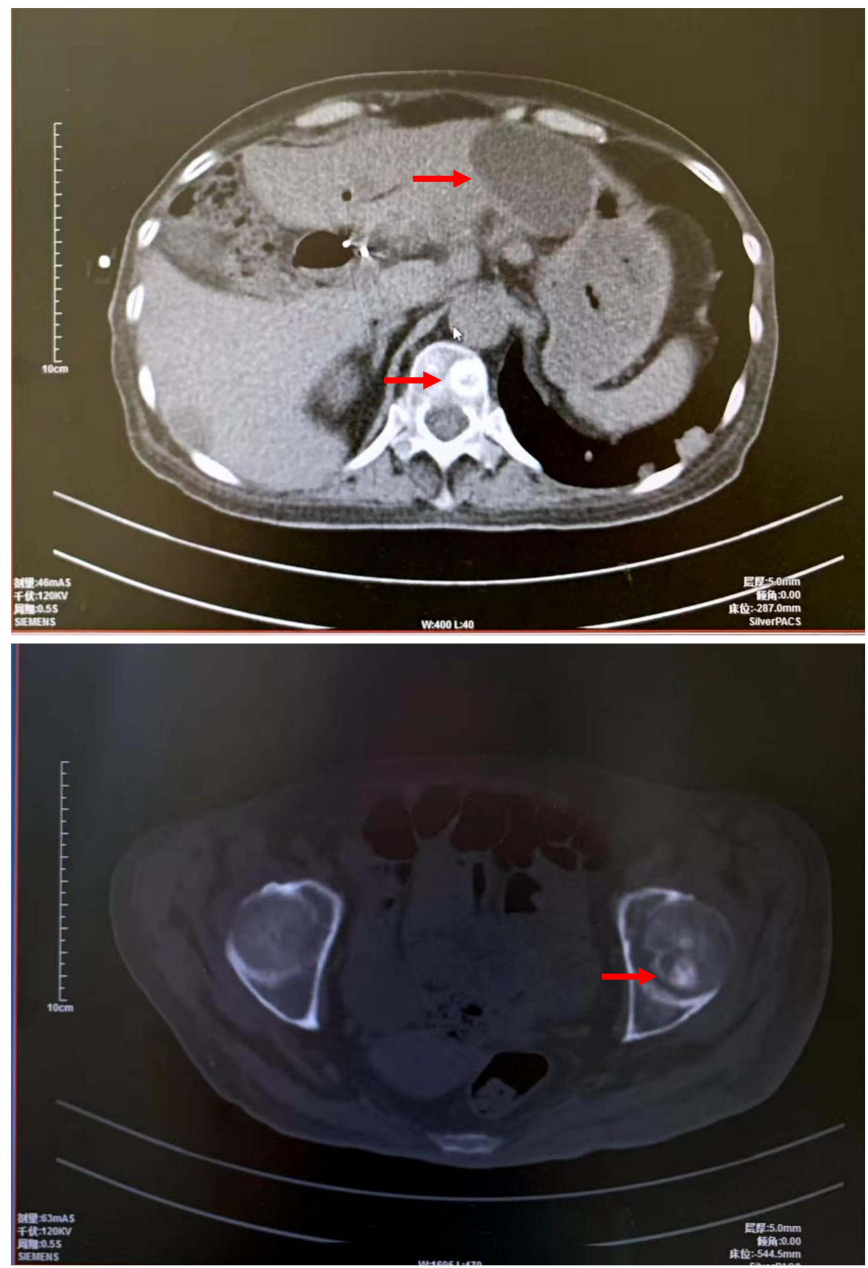

D

Figure 2 Computed tomography (CT) scan of the chest and abdomen. Multiple metastases of the tumor were observed in the lung (A), liver, vertebral body (B), retroperitoneum $(\mathbf{C})$, and caput femoris (D).

\section{Discussion}

Cryptococcus neoformans is an opportunistic pathogen found in soil, trees, decaying wood, fruit, vegetables and bird droppings. ${ }^{11}$ Compared with systemic diseases, PCC is recognized as a distinct clinical entity and rarely reported in patients with normal immune function. Clinical diagnosis of PCC is usually difficult due to its nonspecific skin manifestations characterized by infiltrative lesions confined to the skin and subcutaneous tissue. Culture and histology remain the gold standards for the diagnosis of cryptococcosis. ${ }^{12}$ While no definitive standard treatment protocol exists for PCC, fluconazole and itraconazole are commonly used to treat this condition, with their dose and treatment durations contingent on the patient's immune status and degree of involvement. ${ }^{13,14}$
A treatment duration of 6-12 months is recommended by the Infectious Diseases Society of America (IDSA) for patients without dissemination and nervous system involvement. $^{3}$

There are reports of PCC in patients with lymphoma, leukaemia and rectal adenocarcinoma combined with multiple metastases who were treated with targeted drugs, immunosuppressive agents or radiotherapy. ${ }^{15-17}$ However, to the best of our knowledge, the patient presented here represents the first reported case of PCC with MSCC. The presentation of multiple skin lesions limited to both thighs without systemic dissemination in this patient leads to an initial misdiagnosis of erysipelas, with the antibiotic therapy administered being ineffective. Culture of pus from the infected areas revealed Cryptococcus neoformans. The 

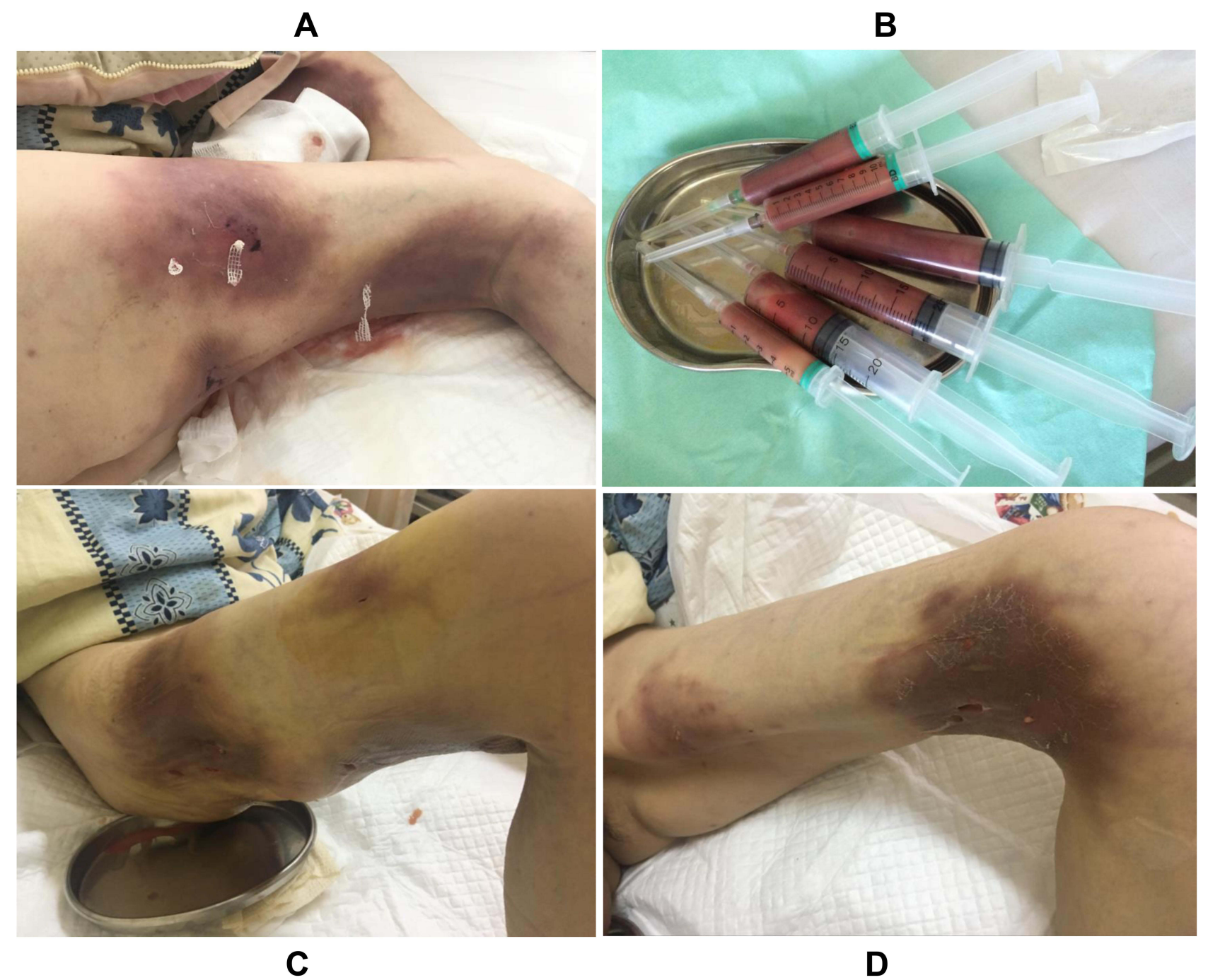

Figure 3 Treatment with cutting the abscesses and lavaging the abscess cavities with fluconazole saline solution (A). A large amount of pus from the abscesses (B). Inflammation within the skin lesions was obviously improved after a week of treatment (C and D).

occurrence of PCC is mainly due to immunodepression as a result of MSCC metastases without treatment. Although the patient succumbed to the progression of the cancer six months after discharge, the topical treatment of fluconazole achieved a favorable outcome with no recurrence of skin lesions. Fluconazole is routinely used as a broad-spectrum antifungal agent in the treatment of cryptococcosis, usually administered orally or intravenously. ${ }^{18}$ In addition to this systemic antifungal agent treatment, the abscesses were incised and the abscess cavities were lavaged with fluconazole saline solution, to reduce the progression of the lesions. Although the patient had terminal cancer with multiple systemic metastases, presenting cachexia, hypoproteinemia and was immunocompromised, which increased her chances of being infected, there was no systemic dissemination due to the infection found at the time of admission.
This case indicates that clinicians should be alerted to the possibility of Cryptococcus neoformans in patients with advanced malignant tumors complicated with multiple skin and soft tissue infections. Skin lesions that do not respond to antibiotics require additional tests to exclude other pathogens, such as fungi. Timely diagnosis and treatment are essential for the recovery of cryptococcosis.

\section{Abbreviations}

MSCC, mediastinal small cell carcinoma; PCC, Primary cutaneous cryptococcosis; HIV, human immunodeficiency virus; CA125, carbohydrate antigen 125; CA199, carbohydrate antigen 199; NSE, neuronspecific enolase; CT, computed tomography; CSF, cerebrospinal fluid; TTE, transthoracic echocardiography; ECG, electrocardiogram. 


\section{Ethical Approval}

The patient's informed consent for publication of the case details including the images was obtained and the study was approved by Ethics Committee of the Second Hospital of Dalian Medical University.

\section{Author Contributions}

All authors have made significant contributions to the work reported, such as data acquisition, analysis, interpretation, drafting, revising, giving final approval of the version to be published, agreeing on the journal and to take responsibility and be accountable for the contents of the article.

\section{Funding}

This work was supported by the Natural Science Foundation of Liaoning Province, China [No.2020-MS -262, No.20180550028].

\section{Disclosure}

The authors declare no conflicts of interest in this work.

\section{References}

1. Gaylord EA, Choy HL, Doering TL. Dangerous Liaisons: interactions of Cryptococcus neoformans with host phagocytes. Pathogens. 2020;9 (11):891. doi:10.3390/pathogens9110891

2. Gushiken AC, Saharia KK, Baddley JW. Cryptococcosis. Infect Dis Clin North Am. 2021;35(2):493-514. doi:10.1016/j.idc.2021.03.012

3. Perfect JR, Dismukes WE, Dromer F, et al. Clinical practice guidelines for the management of cryptococcal disease: 2010 update by the Infectious Diseases Society of America. Clin Infect Dis. 2010;50 (3):291-322. doi:10.1086/649858

4. Christianson JC, Engber W, Andes D. Primary cutaneous cryptococcosis in immunocompetent and immunocompromised hosts. Med Mycol. 2003;41(3):177-188. doi:10.1080/1369378031000137224

5. Marques SA, Bastazini I, Martins ALGP, et al. Primary cutaneous cryptococcosis in Brazil: report of 11 cases in immunocompetent and immunosuppressed patients. Int J Dermatol. 2012;51(7):780-784. doi:10.1111/j.1365-4632.2011.05298.x
6. Longhitano S, Peccerillo F, Odorici G, Bigi L, Pellacani G. An unusual presentation of primary cutaneous cryptococcosis. Dermatol Ther. 2019;32(4):e12942. doi:10.1111/dth.12942

7. Shalom G, Horev A. Primary Cutaneous Cryptococcosis: an Unusual Injection Site Infection. Case Rep Dermatol. 2020;12(2):138-143. doi: $10.1159 / 000508783$

8. Neuville S, Dromer F, Morin O, et al. Primary cutaneous cryptococcosis: a distinct clinical entity. Clin Infect Dis. 2003;36(3):337-347. doi:10.1086/345956

9. Noguchi H, Matsumoto T, Kimura U, Hiruma M, Kusuhara M, Ihn H. Cutaneous Cryptococcosis. Med Mycol J. 2019;60(4):101-107. doi: $10.3314 / \mathrm{mmj} .19 .008$

10. Dai W, Liu M, Zhuang X, Li Q, Wang D. Mediastinal small cell carcinoma: a unique clinical entity? Clin Transl Oncol. 2016;18 (5):515-520. doi:10.1007/s12094-015-1398-4

11. Yu CH, Chen Y, Desjardins CA, et al. Landscape of gene expression variation of natural isolates of Cryptococcus neoformans in response to biologically relevant stresses. Microb Genom. 2020;6(1):e000319. doi:10.1099/mgen.0.000319

12. Du L, Yang Y, Gu J, Chen J, Liao W, Zhu Y. Systemic review of published reports on primary cutaneous cryptococcosis in immunocompetent patients. Mycopathologia. 2015;180(1-2):19-25. doi:10. 1007/s11046-015-9880-7

13. Saag MS, Graybill RJ, Larsen RA, et al. Practice guidelines for the management of cryptococcal disease. Clin Infect Dis. 2000;30 (4):710-718. doi:10.1086/313757

14. Choi H, Kim YI, Na CH, Kim MS, Shin BS. Primary Cutaneous Cryptococcosis in an Older Immunocompetent Patient: a Case Report. Ann Geriatr Med Res. 2020;24(2):148-151. doi:10.4235/agmr.20.0012

15. Peri AM, Rossio R, Tafuri F, et al. Atypical primary cutaneous cryptococcosis during ibrutinib therapy for chronic lymphocytic leukemia. Ann Hematol. 2019;98(12):2847-2849. doi:10.1007/s00 277-019-03837-1

16. Romano C, Taddeucci P, Donati D, Miracco C, Massai L. Primary cutaneous cryptococcosis due to Cryptococcus neoformans in a woman with non-Hodgkin's lymphoma. Acta Derm Venereol. 2001;81(3):220-221. doi:10.1080/000155501750376429

17. Posada C, de la Torre C, González-Sixto B, Cruces MJ. Primary cutaneous cryptococcosis presenting with a sporotrichoid pattern in a cancer patient. Actas Dermosifiliogr. 2009;100(1):78-80. doi:10. 1016/S0001-7310(09)70064-X

18. Izumikawa K, Kakeya H, Sakai F, et al. Executive Summary of JSMM Clinical Practice Guidelines for Diagnosis and Treatment of Cryptococcosis. Med Mycol J. 2020;61(4):61-89. doi:10.3314/ mmj.20.001
Infection and Drug Resistance

\section{Publish your work in this journal}

Infection and Drug Resistance is an international, peer-reviewed openaccess journal that focuses on the optimal treatment of infection (bacterial, fungal and viral) and the development and institution of preventive strategies to minimize the development and spread of resistance. The journal is specifically concerned with the epidemiology of antibiotic resistance and the mechanisms of resistance development and diffusion in both hospitals and the community. The manuscript management system is completely online and includes a very quick and fair peerreview system, which is all easy to use. Visit http://www.dovepress.com/ testimonials.php to read real quotes from published authors. 